\title{
Tako-Tsubo syndrome: A literature review and a case report
}

\author{
Francesco Bartolomucci ${ }^{1}$, Giuseppe Nasso ${ }^{2 *}$, Francesco Cipriani ${ }^{1}$, Giovanni Deluca ${ }^{1}$, Gianluca Sgarra ${ }^{1}$, \\ Carlo D'Agostino ${ }^{3}$, Giuseppe Speziale ${ }^{2}$
}

\author{
${ }^{1}$ Department of Cardiology, ANDRIA Hospital, Andria, Italy \\ ${ }^{2}$ Department of Cardiac Surgery, Anthea Hospital, GVM Care \& Research, Bari, Italy \\ ${ }^{3}$ Division of Cardiology, Intensive Care Unit, “Di Venere” Hospital, Bari, Italy \\ Email: gnasso@libero.it
}

Received 11 April 2012; revised 14 May 2012; accepted 25 May 2012

\begin{abstract}
The Tako-Tsubo syndrome is a rare yet recognized condition characterized by acute but rapidly reversible left ventricular dysfunction in absence of obstructive coronary artery stenosis. Although several factors have been associated with the development of this syndrome, still its etiology remains undefined. We report the case of a 51-year-old woman presenting with dyspnea in whom the diagnosis of TakoTsubo syndrome was posed. We describe the diagnostic modalities and the clinical outcome of the patient. Secondly, the present paper is aimed at providing a review of the current evidence over this topic and at proposing future research directions.
\end{abstract}

Keywords: Tako-Tsubo syndrome; Pathophysiology

\section{INTRODUCTION}

Tako-Tsubo syndrome (TTS), first reported in Japan [1], is a recently described clinical entity also known as "transient apical ballooning syndrome" or "stress cardiomyopathy": this is a type of non-ischemic cardiomyopathy characterized by acute but rapidly reversible left ventricular (LV) dysfunction in absence of obstructive atherosclerotic coronary artery stenosis $($ i.e.: $<\mathrm{o}=50 \% \mathrm{lu}-$ minal narrowing of the epicardial arteries by angiography), even if this could be suggested by the electrocardiographic findings. The etiology of such a syndrome remains unclear, but it is known to be triggered by intense emotional or physical stress or by the exacerbation of concurrent medical conditions (epilepsy, stroke, acute abdomen, septicemia, acute asthma exacerbation, respiratory failure).

\section{CASE REPORT}

We present the case of a 51-year-old woman, without any

${ }^{*}$ Corresponding author. relevant medical history, nor cardiovascular risk factors, who was admitted to our hospital emergency room with progressive dyspnoea. Thoracic pain irradiated to both arms was also present. ECG revealed only a left axis deviation with a normal sinus rhythm and a frequency of 90 beats per minute. Echocardiographic findings showed a normal left ventricular function (Ejection Fraction: $60 \%$ ), relevant right ventricular dilation and dysfunction associated with severe pulmonary hypertension (70 $\mathrm{mmHg}$ ). The subcostal view showed inferior vena caval dilation; a floating thrombus $(35 \times 12 \mathrm{~mm})$ was observed within. The Angio-CT scan confirmed the presence of the thrombus, and demonstrated bilateral, multiple pulmonary artery circulation abnormalities. Our patient underwent anticoagulant therapy (heparin) and R-Tpa thrombolytic treatment, with an immediate improvement of symptoms and clinical picture. Two days later, sudden thoracic pain appeared: there were negative $T$-waves in V1 - V6 and the echocardiogram showed severe left ventricular dysfunction (EF: 25\%) and cardiac wall motion abnormalities involving the left apical and midventricular myocardial segments (Figure 1). Coronary angiography was performed: no epicardial coronary stenosis was evident, while ventriculography confirmed the echocardiographic findings. Twenty-four hours after her admission, our patient underwent a trans-thoracic color Doppler echocardiogram to assess the Coronary Flow Reserve (CFR): this was measured targeting the distal portion of the left anterior descending artery with the ultrasound probe, while injecting adenosine $(0.14 \mathrm{mg} / \mathrm{Kg} / \mathrm{min}$ in 2 minutes). The result was 2.3. Four hours later, a myocardial regional perfusion assessment was performed, using Sono-Vue as a contrast agent; the perfusion was found to be normal in all affected districts, except for a small sub-endocardial apical region. On these bases, a diagnosis of Tako-Tsubo syndrome was made. One month after discharge, the ECG was normal and so was the echocardiogram, which showed a normal ventricular 


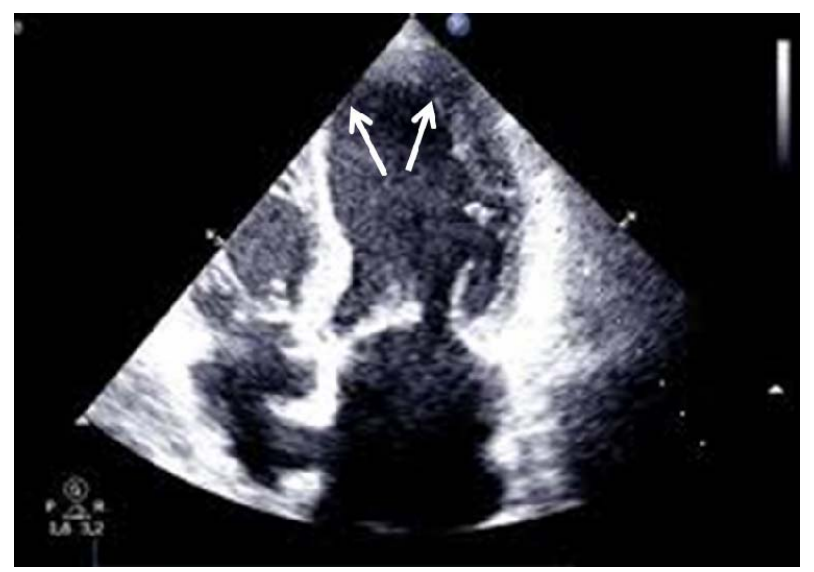

Figure 1. Transthoracic echocardiography: apical fourchamber view showing apical ballooning (white arrows).

wall motion and EF. The patient's response to the adenosine was improved: the recorded flow speed was three times faster than the baseline. The echocontrastographic assessment demonstrated a complete normalization of the perfusion in all myocardial areas.

\section{CASE DISCUSSION}

Tako-Tsubo syndrome (TTS) is a rare, newly described syndrome characterized by a transient, acute-onset systolic dysfunction without epicardial coronary artery disease. This syndrome was originally documented in Japanese population, but subsequent observations found out that no ethnic predilection exists [2]. Although it has been reported a familial association for these disorders, genetic studies did not find any genetic basis for such a correlation yet [3]. TTS involves about $1 \%$ of all suspect cases of acute coronary syndrome [4]; it has been previously described in postmenopausal women, but literature reports this entity also in younger adults and children [5-7]. This form of ventricular stunning usually involves the distal portion of the left ventricle and the most frequently recognized triggering factor is the emotional or physical stress. However, TTS do not always appear to have an identifiable trigger factor. The death of a close relative is one of the most recognized stressors [8], but in our case the presence of a thrombus in the patient's inferior vena cava and the multiple pulmonary circulation abnormalities could reasonably be the trigger event for the subsequent TTS.

Additionally, the present clinical case underscores the importance of maintaining an high level of suspicion for the diagnosis of TTS, since the clinical picture at presentation may be very unspecific. In fact, our patient presented with a history of recently aggravating dyspnea. Other symptoms, such as chest pain, could raise the suspicion of acute coronary syndrome. As such, the picture may be even more misleading since ECG abnormalities mimicking acute coronary syndrome may be evident, as in our case. The diagnostic criteria to properly recognize TTS include the following: the patients usually suffer from chest pain, often of in crescendo (68\%) (as we experienced in our patient), and dyspnoea (18\%). A ST segment elevation is frequent $(81 \%)$, followed by $\mathrm{T}$ wave abnormalities (64\%) and Q-waves (31\%) [6], especially in leads $V_{3}$ to $V_{6}$ [9]. A mild troponine elevation is also frequent [10]. These ECG abnormalities are indistinguishable from those of acute coronary syndrome due to epicardial coronary stenosis, but in TTS such abnormalities are reversible: even the Q-waves usually resolve within 30 days from the acute episode. They are believed to represent local Purkinje fiber death or dysfunction, as testified by the lack of hyperenhancement in MRI [11]. The possibility of such misinterpretation of the clinical findings should not suggest to avoid coronary angiography when the suspicion of TTS is present. To exclude the presence of significant epicardial coronary lesions should remain an important clinical step in all cases with presumption of acute coronary syndrome. In our patient, the diagnosis was based mainly on echocardiography and on the clinical course. The various echocardiography techniques represent the most reliable diagnostic modality for TTS; in fact, even MRI is not believed to be more informative than echocardiography. The finding of late gadolinium enhancement (LGE) as a sign of myocardial damage is rarely seen in MRI of patients with TTS: Sharkey et al. reported a large series of 22 cases, with only one presenting LGE [8]. Transient cardiac wall motion abnormalities involving apical or midventricular myocardial segments (typical form) or mid basal segments (atypical form) must be considered too and they are extended beyond a single vessel territory. The involvement of the right ventricular apex and of the free wall is also common, and no trace of significant atherosclerotic coronary artery disease should be present, neither of other concurrent medical conditions (e.g. pheochromocytoma, epilepsy, stroke, acute abdomen, intracranial bleeding). Many Authors consider stroke and pheochromocytoma as authentic "stress cardiomyopathies"; especially the latter may cause typical as well as atypical TTS (inverted Tako-Tsubo). In-Hospital mortality of TTS is quite low (1\% to $3 \%)$ : associated complications include hemodynamic instability, atrial and ventricular arrhythmias; heart failure with pulmonary edema and cardiogenic shock can occur in a minority of patients. Transient right ventricular dysfunction can be a common complication of TTS; the right ventricular involvement can even be the only sign of TTS. The formation of left or right ventricular thrombi is also possible, due to the contraction impairments, which may even generate embolisms [12]. The prognosis for these patients is quite good with an almost complete resolution of the left ventricle impair- 
ment in a few days to weeks. For these reasons, once the nature of our patient's problems was clear, we discharged her after 10 days from recovery, programming a follow up regimen, which allowed us to verify a typical normalization of the parameters after one month.

\section{LITERATURE REVIEW}

The exact etiology of TTS remains unclear: histological findings on endomyocardial biopsy show mononuclear infiltrates, focal myocytolysis and contraction band necrosis, without any evidence of myocarditis [13]. At first, multivessel coronary spasm was suggested to be the main cause of the disease [14]; nonetheless, further studies based on provocative tests, demonstrated that such an hypothesis could not be considered true. In one study multi- or single-vessel spasm occurred in only a few patients (21\%: 10 of 48) after the administration of ergonovine or acetylcholine [15] and in one other only one case of vasospasm was reported in all the tested patients (14\%) [16]. Conflicting results have been reported in the literature concerning microvascular dysfunction, because of the nuclear imaging findings of decreased myocardial perfusion using single photon emission computed tomography (SPECT): the low perfusion assessed in absence of coronary obstructions can be interpreted as a proof of impaired coronary microcirculation [17]. On the other hand, Abe et al. [16] did not consider the scintigraphic abnormalities found in their TTS patients as perfusion abnormalities. This could be attributed to the mechanism of uptake of Tc-tetrofosmin by myocytes. Such process occurs via a metabolism-dependent pathway and its subcellular localization is in mitochondria. Thus, they suggested that the scintigraphic abnormality was caused by abnormalities in the mitochondria, rather than by coronary microcirculatory damage. Excessive sympathetic stimulation and catecholamine release seem now to be crucial in the pathogenesis of TTS $[18,19]$. Ueyama et al. [20] induced transient left ventricle ballooning in rats exposed to emotional stress, but nothing happened after a pre-treatment with $\alpha$ - and $\beta$-blockers. Akashi et al. [21] performed iodine-123-meta-iodobenzylguanidine $\left({ }^{123} \mathrm{I}\right.$ MIBG) serial myocardial scintigraphy in eight patients during the index admission and at 3 months follow-up: by comparing the heart-mediastinum ratios and the higher radioisotope washout in the initial than in the follow-up scans, they argued that neurogenic myocardial stunning and hypersympathetic activity can be important mechanisms for the genesis of TTS. By ${ }^{18} \mathrm{~F}$-fluorodeoxyglucose positron emission tomography imaging (PET) a reduction in regional glucose uptake was demonstrated, thus corroborating the hypothesis involving myocardial stunning and microcircolatory damage [5]. Cardiac magnetic resonance imaging (MRI), in spite of the use of myocardial contrast-enhancement agents to prove the hyperenhancement and the non-viability of myocardium, has failed to document myocardial necrosis, nevertheless the almost universal finding of mild troponin elevation in TTS. Gadolinium hyperenhancement is noticeable only in the acute phase, due to edema and flogosis [22]. There is no late myocardial gadolinium enhancement, as it is seen in myocarditis or in embolic infarction, which denotes the absence of detectable ischemic myocardial necrosis in most TTS patients [23,24]. The high spatial resolution of contrast-enhanced MRI will leave no room for any further doubt. However, a few patients show small areas of left ventricular subendocardial delayed hyperenhancement confined to the left ventricular apex [25]. Recently Rolf et al. [26] described the immunohistological basis of the late gadolinium enhancement in TTS, by combining the results of laboratory findings, immunohistochemistry and electronic microscopy performed on tissue samples of endomyocardial biopsies. Late gadolinium enhancement in TTS is reasonably due to transient post-acute fibrosis which presence is limited to the extracellular and interstitial space, mostly for a limited time. Myocardial contrast echocardiography (MCE) is up to day an attractive solution to assess the TTS, because of its reproducibility, its low costs and since it can be performed at the patients' bedside. The latter represents one ideal condition to analyse the myocardial perfusion. This newly developed technique utilizes gas-filled microbubbles causing backscattering ultrasound echoes due to their very small radius $(<5 \mu \mathrm{m})$. The rheology of the microbubbles is similar to that of erythrocytes, thus allowing a check of the cardiac perfusion and of the integrity of the microcirculation. After contrast injection, a high-energy ultrasound pulse must be delivered to destroy these microbubbles once they reach the myocardium; then, low mechanical index images can be acquired and the changes over time, which express the microcirculatory replenishment time, allow to calculate the erythrocyte velocity. Pulsed-wave MCE has been reported in literature only few times and allows to document the disruption of microvasculature in TTS, thus showing not only the apical absence of perfusion in the immediate circumstances, but also the complete recovery within 72 hours [27]. This is congruent with arterial vasoconstriction [28] and a significant loss of the capillary bed volume. Nef et al. [29] found out that the overstimulation of $\beta$-adrenoceptors by catecholamine can precipitate intracellular calcium overload and caus myocyte damage. Galiuto et al. [30] reported that in each of their 15 patients the perfusion defect within the dysfunctional myocardial region (which was transmural in 11 of 15) decreased in extent after adenosine infusion, and immediately returned to baseline conditions after cessation of the administration. At the 1 month follow-up their conditions significantly improved if compared to base- 
line. A complete normalization was observed in 13 out of 15; conversely, the 15 patients enrolled in the STEMI group did not show such an improvement over time, neither after administration of adenosine, nor at the1 month follow-up. These facts demonstrated for the first time that reversible coronary microvascular impairment caused by vasoconstriction is the common pathophysiological determinant of this syndrome [30]. The left ventricle apex is highly trabeculated, thus increasing its surface area exposed to circulating cathecholamines [31]. Moreover, the severity and extent of perfusion impairments in TTS match the abnormalities in segmental contractile functions and similarly improve during the follow-up. The improvements in Coronary Flow Reserve (CFR) as assessed via trans thoracic echocardiography, parallel the improvements in regional left ventricle contractile function, even though these events do not occur contemporarily. CFR reaches normal values in the majority of patients at discharge, while the Wall Motion Score Index (WMSI) keeps on improving until reaching a complete normalization within 6 months [32]. The management and treatment options for a patient with TTS are similar than patients affected by acute myocardial infarction (MI), at least until the differential diagnosis has been established. However, this disorder can be easily misderdiagnosed; additionally, because of the small number of cases reported in literature, there is no consensus regarding the appropriate therapy for TTS. No initial sign, symptom or ECG finding really differentiates TTS from MI, usually resembling STEMI; therefore one should follow the usual protocols for the management of the acute coronary syndrome. Hypotension may occur In the acute phase of TTS [33]: this is the consequence of left ventricular dysfunction, or of left ventricular outflow tract obstruction and systolic anterior motion of the mitral valve.

The ideal treatment for TTS is the placement of an Intra Aortic Balloon Pump (IABP), rather than using inotropes (which may exacerbate the left ventricular impairment, and add their effect to the already existing catecholamine excess) [33]. If the left ventricular obstruction is the predominant feature, then intravenous short-acting $\beta$-blockers should be administered to reduce contractility and slightly widen the left ventricular cavity [33]. In these cases, vasodilators such as nitrates should be avoided, due to the risk is to worsen the obstruction itself. The inpatient care management includes continuous telemetry monitoring, administration of drugs such as $\beta$-blockers (when hemodynamics allow), aspirin, anticoagulants with direct thrombin inhibition or inhibiting glycoprotein IIb/IIIa receptor, nitrates and diuretics and all the supportive care required in a conservative approach to avoid complications. The treatment therefore continues to be the same as MI. Patients with left ven- tricular clot formation, which occurs in $5 \%$ of cases of TTS, require anticoagulation [10]. Akashi et al. [34] noted complete reversal of contractile abnormalities and recovery with no treatment. When no coronary artery or peripheral vascular disease has been assessed, aspirin can be discontinued, while $\beta$-blockers may be continued in the long-term to protect against catecholamine sensitivity, which may predispose to TTS [35]. After discharge and full normalization, annual clinical follow-up is advised, because the long-term effects and the natural history of TTS are actually unknown [36].

\section{CLASSIFICATION}

Shimizu et al. [37] recently collected numerous cases of TTS and proposed a classification on the basis of the various ballooning patterns.

1) Takotsubo type: apical akinesis and basal hyperkinesia.

2) Reverse takotsubo: basal akinesis and apical hyperkinesia.

3) Mid ventricular type: mid ventricular ballooning accompanied by basal and apical hyperkinesia.

4) Localised type: any other segmental LV ballooning with clinical characteristics of takotsubo-like LV dysfunction.

5) Evolutive pattern: it progressively involves the left ventricle from its middle portion to the apex.

Right ventricular impairment is not classified here, since the consequent complications must be considered separately.

\section{CONCLUSION}

The available evidence indicate that, even if TTS is still an underrated condition, effective therapeutic strategies can be put in action after a swift and correct differential diagnosis. For instance, the expandingaccess to angiography services will lead to earlier recognition and less inappropriate thrombolysis. Fortunately, TTS is a selflimiting disease in the majority of cases. In fact, it is most likely caused by a reversible myocardial dysfunction, shows the features of myocardial stunning, most likely due to excessive sympathetic stimulation.

\section{REFERENCES}

[1] Satoh, H.T.H., Tateishi, H., Uchida, T., et al. (1990) Takotsubotype cardiomyopathy due to multivessel spasm. In: Kodama, K., Haze, K. and Hon, M., Eds., Clinical Aspect of Myocardial Injury: From Ischemia to Heart Failure (in Japanese). Kagakuhyouronsya Co., Tokyo, 56-64.

[2] Desmet, W., Adriaenssens, B. and Dens, J.A. (2003) Apical ballooning of the left ventricle: First series in white patients. Heart, 89, 1027-1031.

doi:10.1136/heart.89.9.1027 
[3] Kumar, G., Holmes, D. Jr. and Prasad, A. (2010) Familial apical ballooning syndrome (Takotsubo-cardiomyopathy). International Journal of Cardiology, 144, 444-445. doi:10.1016/j.ijcard.2009.03.078

[4] Bybee, K.A., Murphy, J., Prasad, A., Wright, R.S., Lerman, A., Rihal, C.S., et al. (2006) Acute impairment of regional myocardial glucose uptake in the apical ballooning (takotsubo) syndrome. Journal of Nuclear Cardiology, 13, 244-250.

[5] Maruyama, S., Nomura, Y., Fukushige, T., Eguchi, T., Nishi, J., Yoshinaga, M., et al. (2006) Suspected takotsubo cardiomyopathy caused by withdrawal of bupirenorphine in a child. Circulation Journal, 70, 509-511. doi:10.1253/circj.70.509

[6] Gianni, M., Dentali, F., Grandi, A.M., Sumner, G., Hiralal, R. and Lonn, E. (2006) Apical ballooning syndrome or takotsubo cardiomyopathy: A systematic review. European Heart Journal, 27, 1523-1529. doi:10.1093/eurheartj/ehl032

[7] Reuss, C.S., Lester, S.J., Hurst, R.T., Askew, J.W., Nager, P., Lusk, J., et al. (2007) Isolated left ventricular basal ballooning phenotype of transient cardiomyopathy in young women. American Journal of Cardiology, 99, 14511453. doi:10.1016/j.amjcard.2006.12.078

[8] Sharkey, S.W., Lesser, J.R., Zenovich, A.G., et al. (2005) Acute and reversible cardiomyopathy provoked by stress in women from the United States. Circulation, 111, 472479. doi:10.1161/01.CIR.0000153801.51470.EB

[9] Kurisu, S., Inoue, I., Kawagoe, T., Ishihara, M., Shimatani, Y., Nakamura, S., et al. (2004) Time course of electrocardiographic changes in patients with Tako-Tsubo syndrome: Comparison with acute myocardial infarction with minimal enzymatic release. Circulation Journal, 68, 77-81. doi:10.1253/circj.68.77

[10] Pilgrim, T.M. and Wyss, T.R. (2008) Takotsubo-cardiomyopathy or transient left ventricular apical ballooning syndrome: A systematic review. International Journal of Cardiology, 124, 283-292.

doi:10.1016/j.ijcard.2007.07.002

[11] Sztajzel, J. and Urban, P. (2000) Early and late Q wave regression in the setting of acute myocardial infarction. Heart, 83, 708-710. doi:10.1136/heart.83.6.708

[12] Kurisu, S., Inoue, I., Kawagoe, T., Ishihara, M., et al. (2011) Incidence and treatment of left ventricular apical thrombosis in Tako Tsubo cardiomiopathy. International Journal of Cardiology, 146, e58-e60. doi:10.1016/j.ijcard.2008.12.208

[13] Wittstein, I.S., Thiemann, D.R., Lima, J.A., Baughman, K.L., Schulman, S.P., Gerstenblith, G., et al. (2005) Neurohumoral features of myocardial stunning due to sudden emotional stress. The New England Journal of Medicine, 352, 539-548. doi:10.1056/NEJMoa043046

[14] Dote, K., Sato, H., Tateishi, H., Uchida, T. and Ishihara, M. (1991) Myocardial stunning due to simultaneous multivessel spasms: A review of five cases. Journal of Cardiology, 21, 203-214.

[15] Kurisu, S., Sato, H., Kawagoe, T., Ishihara, M., Shimatani, Y., Nishioka, K., et al. (2002) Tako-Tsubo-like left ven- tricular dysfunction with ST-segment elevation: A novel cardiac syndrome mimicking acute myocardial infarction. American Heart Journal, 143, 448-455. doi:10.1067/mhj.2002.120403

[16] Abe, Y., Kondo, M., Matsuoka, R., Araki, M., Dohyama, K. and Tanio, H. (2003) Assessment of clinical features in transient left ventricular apical ballooning. Journal of the American College of Cardiology, 41, 737-742. doi:10.1016/S0735-1097(02)02925-X

[17] Ito, K., Sugihara, H., Katoh, S., Azuma, A. and Nakagawa, M. (2003) Assessment of Takotsubo (ampulla) cardiomyopathy using 99m Tc-Tetrofosmin myocardial SPECT-comparision with acute coronary syndrome. $A n$ nals of Nuclear Medicine, 17, 115-122. doi:10.1007/BF02988449

[18] Scanzerla, P., Alioto, G., Funaro, A., Passaretti, B., Borghini, E. and Guglielmetto, S. (2008) Transthoracic Doppler ultrasound flow reserve evaluation: Preliminary insights into pathophysiology of Takotsubo cardiomyopathy. Journal of Cardiovascular Medicine, 12, 1229-1234. doi:10.2459/JCM.0b013e328313e890

[19] Lyon, A., Rees, P.S.C., Prasad, S., Poole-Wilson, P.A. and Harding, S.E. (2008) Stress (Takotsubo) cardiomyopathy-A novel pathophysiological hypothesis to explain catecholamine-induced acute myocardial stunning. $\mathrm{Na}$ ture Clinical Practice, 5, 22-29. doi:10.1038/ncpcardio1066

[20] Ueyama, T., Kasamatsu, K., Hano, T., Yamamoto, K., Tsuruo, Y. and Nishio, I. (2002) Emotional stress induces transient left ventricular hypocontraction in the rat via activation of cardiac adrenoceptors: A possible animal model of 'Tako-Tsubo' cardiomyopathy. Circulation Journal, 66, 712-713. doi:10.1253/circj.66.712

[21] Akashi, Y.J., Nakazawa, K., Sakakibara, M., Miyake, F., Musha, H. and Sasaka, K. (2004) ${ }^{123}$ I-MIBG myocardial scintigraphy in patients with 'takotsubo' cardiomyopathy. Journal of Nuclear Medicine, 45, 1121-1127.

[22] Avegliano, G., Huguet, M., Costabel, P.J., Ronderos, R., Bijnens, B., Kuschnir, P., et al. (2011) Morphologic pattern of late Gadolinium Enhancement in Tako Tsubo Cardiomyopathy detected by early Cardiovascular Magnetic Resonance. Clinical Cardiology, 34, 178-182. doi:10.1002/clc.20877

[23] Mitchell, J.H., Hadden, T.B., Wilson, J.M., Achari, A., Muthupillai, R. and Flamm, S.D. (2007) Clinical features and usefulness of cardiac magnetic resonance imaging in assessing myocardial viability and prognosis in Takotsubo cardiomyopathy (transient left ventricular apical ballooning syndrome). American Journal of Cardiology, 100, 296-301. doi:10.1016/j.amjcard.2007.02.091

[24] Nanda, S., Bhatt, S.P., Dale, T.H., Kurowki, V., Burgdorf, C. and Radke, P.W. (2008) Tako tsubo cardiomyopathy: An insight into pathogenesis. Chest, 133, 583-584. doi:10.1378/chest.07-1745

[25] Sharkey, S.W., Lesser, J.R., Zenovich, A.G., Maron, M.S., Lindberg, J., Longe, T.F. and Maron, B.J. (2005) Acute and reversible cardiomyopathy provoked by stress in women from the United States. Circulation, 111, 472-479. doi:10.1161/01.CIR.0000153801.51470.EB 
[26] Rolf, A., Nef, M.H., Möllmann, H., Troidl, C., Voss, S., Conradi, G., et al. (2009) Immunohistological basis of the late gadolinium enhancement phenomenon in Tako Tsubo cardiomyopathy. European Heart Journal, 30, 1635-1642. doi:10.1093/eurheartj/ehp140

[27] Shrikanth, P.Y., et al. (2005) Tako Tsubo cardiomyopathy (transient left ventricular apical ballooning): Case report of a myocardial perfusion echocardiogram study. Journal of the American Society of Echocardiography, 18, e10e14.

[28] Wei, K. and Kaul, S. (2004) The coronary microcirculation in health and disease. Cardiology Clinics, 22, 221231. doi:10.1016/j.ccl.2004.02.005

[29] Nef, H.M., Möllmann, H., Troidl, C., Kostin, S., et al. (2009) Abnormalities in intracellular $\mathrm{Ca} 2+$ regulation contribute to the pathomechanism of Tako-Tsubo cardiomyopathy. European Heart Journal, 30, 2155-2164. doi:10.1093/eurheartj/ehp240

[30] Galiuto, L., Ranieri De Caterina, A., Porfidia, A., Paraggio, L., et al. (2010) Reversible coronary microvascular dysfunction: A common pathogenetic mechanism in apical ballooning or Tako-Tsubo syndrome. European Heart Journal, 31, 1319-1327. doi:10.1093/eurheartj/ehq039

[31] Bielecka-Dabrowa, A., Mikhailidis, D.P., Hannam, S., et al. (2010) Takotsubo-cardiomyopathy-The current state of knowledge. International Journal of Cardiology, 142, 120-125. doi:10.1016/j.ijcard.2009.11.040
[32] Rigo, F., Sicari, R., Citro, R., et al. (2009) Diffuse, marked, reversible impairment in coronary microcirculation in stress cardiomyopathy: A doppler transthoracic echo study. Annals of Medicine, 41, 462-470. doi:10.1080/07853890903022793

[33] Hurst, R.T., Prasad, A., Askew, J.W., et al. (2010) Takotsubo Cardiomyopathy: A unique cardiomyopathy with variable ventricular morphology. JACC: Cardiovascular Imaging, 3, 641-649. doi:10.1016/j.jcmg.2010.01.009

[34] Akashi, Y.J., Nakazawa, K., Sakakibara, M., et al. (2003) The clinical features of takotsubo cardiomyopathy. QJM: An International Journal of Medicine, 96, 563-573. doi:10.1093/qjmed/hcg096

[35] Bybee, K.A., Kara, T., Prasad, A., et al. (2004) Systematic review: Transient left ventricular apical ballooning: A syndrome that mimics ST-segment elevation myocardial infarction. Annals of Internal Medicine, 141, 858-865.

[36] Prasad, A., Lerman, A. and Rihal, C.S. (2008) Apical ballooning syndrome (Tako-Tsubo or stress cardiomyopathy): A mimic of acute myocardial infarction. American Heart Journal, 155, 408-417. doi:10.1016/j.ahj.2007.11.008

[37] Shimizu, M., Kato, Y., Masai, H., Shima, T., et al. (2006) Recurrent episodes of takotsubo-like transient left ventricular ballooning occurring in different regions: A case report. Journal of Cardiology, 48, 101-107. 